\title{
Estudiantes a distancia en regiones agrícolas y costeras de Costa Rica: características socio-demográficas, preferencias y acceso a tecnologías
}

\author{
Suzanne Soto Soto \& Carolina Tencio Blanco \\ Programa de Apoyo Curricular y Evaluación de los Aprendizajes, Universidad Estatal a Distancia (UNED), Mercedes de Montes de Oca, San
}

José-Costa Rica; ssoto@uned.ac.cr, ctencio@uned.ac.cr

Recibido 07-VIII-2017 • Corregido 09-XI-2017 • Aceptado 22-XI-2017

\begin{abstract}
Distance students in agricultural and coastal regions of Costa Rica: socio-demographic characteristics, preferences and access to technologies. Costa Rica is one of the Latin American countries with the longest history in distance education, through the Distance State University, UNED, so it is of general interest to know its students and their relationship with technology and education. To assess these characteristics, we applied questionnaires to 66 students from an agricultural and livestock community (Sarapiquí) and 216 from a port community (Puntarenas). They reported a good level of access to electronic devices and Internet; prefer hybrid courses and like the coexistence of varied media and didactic materials. Even though they have a reduced participation in the groups that UNED offers them, they consider participation to be important in the process of professional formation.
\end{abstract}

Key words: distance education, student profile, information and communication technologies, student participation and communication, media and didactic materials
RESUMEN: Costa Rica es uno de los países latinoamericanos con mayor historia en educación a distancia, mediante la Universidad Estatal a Distancia, UNED, por lo que es de interés general conocer a sus estudiantes y la relación que tienen con la tecnología y la educación. Para conocer estas características, aplicamos cuestionarios a 66 estudiantes de una comunidad agropecuaria (Sarapiquí) y 216 de una comunidad portuaria (Puntarenas). Estos Reportaron buen nivel de acceso a dispositivos electrónicos e Internet, prefieren los cursos híbridos y la coexistencia de medios y materiales didácticos; y aunque participan poco en los grupos que la UNED ofrece, lo consideran de importancia en el proceso de formación profesional.

Palabras clave: educación a distancia, perfil estudiantil, tecnologías de información y comunicación, participación y comunicación estudiantil, medios y materiales didácticos
La Educación a Distancia ha tenido una evolución, que se evidencia en el énfasis teórico que décadas atrás se tenía al caracterizarla o definirla más desde aspectos de carácter sociodemográfico, que de aspectos, como es en la actualidad, transaccionales y comunicacionales (García, 2014). Sin embargo, es necesario tenerlas presentes, ya que son condiciones por las que algunas personas encuentran en la modalidad a distancia, una opción para optar por estudios universitarios. A este conjunto de características, las llamaremos "perfil".

Los cambios sociales denotan modificaciones en los perfiles estudiantiles universitarios, configurando "una nueva estructura del colectivo estudiantil" (Soler, 2013, p.14). Edades, condiciones y posibilidades, que varían de un contexto a otro, implican diferencias en cuanto a intereses, ocupaciones, preocupaciones, entre otros. Al plantear la oferta académica, las instituciones deben conocerla, con el fin de mantener como centro de su quehacer al estudiante (García, 2012).

Es raro el estudiante dedicado en su totalidad al estudio y con una participación activa en la institución (Soler 2013), una mayoría dedica tiempo parcial al estudio, para dedicarse a asuntos laborales y de otra índole, por lo que se reduce la vinculación de este con la universidad (Soler 2013).

La población estudiantil de la Universidad Estatal a Distancia de Costa Rica (UNED) ha sido objeto de investigación a través de los años, en un deseo de mantener actualidad con las tendencias mundiales en educación a distancia y en aquellos elementos que la pueden complementar e impulsar en su búsqueda por el mejoramiento y la calidad. 
En un estudio realizado por Méndez, Monge y Montero (2000) se da seguimiento a investigaciones realizadas por Cruz (1996, 1997, 1998), Rivas (1999), Méndez (1999) y Monge (1999), entre otros, donde se destaca una evolución en la aceptación y en la integración de las tecnologías de la información, tanto como recurso que ofrece la universidad, como una posibilidad de escogencia por parte de la población estudiantil.

La presente investigación gira en torno a variables que permiten evidenciar aspectos sociodemográficos y académicos, a indicar: la tenencia de dispositivos electrónicos y su acceso a internet; la preferencia en cuanto a los recursos, medios y materiales didácticos según su experiencia como estudiante UNED y la vinculación que esta población en específico presenta respecto a la institución en términos de participación y comunicación con la institución y sus pares. El Apéndice Digital 1 presenta información adicional sobre estudios previos.

\section{MÉTODOS}

Hicimos la investigación en dos Centros Universitarios (CEU), Sarapiquí y Puntarenas, consideramos población de estudiantes regulares que matricularon en el año 2014, de carreras en los diferentes niveles académicos de pregrado y grado (por lo que no se incluye estudiantes de extensión), con al menos dos cuatrimestres matriculados. Esto permitió tener una población con experiencia en la modalidad de educación a distancia en la UNED.

Decidimos no realizar una selección aleatoria de estudiantes, ya que podrían presentarse dificultades para obtener el número de encuestas requerido. Por ello, la consulta fue enviada a todos(as) los(as) estudiantes de cada CEU (2014), con el propósito de obtener al menos los tamaños mínimos de muestra calculados previamente, aproximándose a un enfoque de muestreo por cuota.

En el caso del CEU de Sarapiquí se obtuvo una población de 158 estudiantes inscritos a lo largo de los tres cuatrimestres y en el caso de Puntarenas 547 estudiantes (Cuadro 1).

Enviamos por Lime Survey un cuestionario de 44 preguntas en total, doce de ellas condicionadas, por lo que la cantidad de preguntas que debió responder cada persona varió.

El cuestionario y su contenido fue validado con expertos (Coordinadora Programa de Orientación y Atención Psicoeducativa de la Dirección de asuntos estudiantiles (DAES), especialista en estadística destacada de la Vicerrectoría de Investigación, Vicerrectora Académica y Directora de Centros Universitarios, Encargado de la Cátedra de Estadística de la UNED, Asesoras curriculares del PACE, Administradoras de los CEU de las poblaciones en estudio). Además, como parte de este proceso, se coordinó con un grupo de seis estudiantes, de diferentes CEU, a quienes se convocó en una sesión para discutir y mejorar la encuesta.

\section{RESULTADOS}

Para efectos de la presentación de los porcentajes de ambos CEU, se indican los de Puntarenas y Sarapiquí respectivamente.

Características sociodemográficas: La población presenta edades entre los 18 y 60 años, siendo los jóvenes y jóvenes adultos el grupo más representativo con más del $70 \%$ y $80 \%$, con una edad promedio de 29 años. Es oportuno indicar que en el caso de Costa Rica (La Gaceta, 2002), la persona joven se delimita por el rango de edad entre los 12 y 35 años (adolescentes, jóvenes y jóvenes adultos) y la Política Pública de la Persona Joven, categoriza en los rangos de edades de 18 a 24 años a los jóvenes y jóvenes adultos entre edades de 25 a 35 años.

Más de la mitad de la población indica un estado civil soltero y no trabajar; en su mayoría mujeres (70\% y 68\%).

El $50 \%$ y $41 \%$ de la población ha interrumpido sus estudios y de esta el $70 \%$ en más de dos periodos académicos. En cuanto a las razones se destacan aquellas de tipo económicas seguidas por las de índole laboral y familiar.

La población indica haber considerado a la UNED como la institución para su formación profesional, por la posibilidad que esta brinda para estudiar y trabajar al mismo tiempo además para organizar el tiempo, el espacio de acuerdo a las preferencias y necesidades y como tercer lugar el costo económico accesible.

CUADRO 1

Tamaño de la población, tamaño de muestra y número de encuestas efectivas en Sarapiquí y Puntarenas

\begin{tabular}{lccc} 
Centro Universitario & Tamaño de la población & Tamaño mínimo de muestra & Encuestas efectivas \\
Sarapiquí & 158 & 63 & 66 \\
Puntarenas & 547 & 189 & 216 \\
\hline
\end{tabular}


Tenencia de dispositivos electrónicos y el acceso y tiempo invertido en internet: Más de la mitad de la población posee dos dispositivos electrónicos, teléfono celular y computadora portátil, con acceso a internet. La mayoría de la población que posee dispositivos electrónicos sí utiliza internet en estos.

Los lugares en donde se accede internet para asuntos académicos con mayor frecuencia, son en orden de prioridad, "la casa" que se constituye como el lugar de mayor frecuencia, seguido "del trabajo o la casa de familiar o amigo". Asimismo hay una población que utiliza la herramienta también desde "los café internet" y el CEU, siendo este último el lugar menos representativo para la población.

La mayoría de la población usa internet en promedio 2 horas al día para asuntos de diversa índole (académico, recreativo, personal y social).

Preferencia de la modalidad de las asignaturas: Más de la mitad de la población tiene experiencia en las tres modalidades que ofrece la UNED, a saber: regulares (sin componentes virtuales) híbridas (integra componentes virtuales y de asignaturas regulares de la educación a distancia) y virtuales (todos los procesos para la enseñanza y el aprendizaje se llevan a cabo en el entorno virtual), lo que requiere de parte de la población dispositivos y acceso a internet, para optar por las modalidades de asignaturas con al menos algún componente virtual.

La población (más del 70\%) prefiere aquellas asignaturas con modalidad virtual o con algún componente virtual. En cuanto a la preferencia de la modalidad híbrida y virtual, prevalecen aquellas que tienen relación con la flexibilidad del componente virtual en cuanto a "Ia atención y horarios de entrega de tareas o participación de actividades varias que la modalidad permite en el proceso de enseñanza y aprendizaje", seguida de otras razones que se relacionan con las condiciones que presenta la población, en cuanto a tenencia de dispositivos y acceso a internet, además de la facilidad del uso de algunas herramientas tecnológicas.

En cuanto a la preferencia de la modalidad regular, se mencionan razones que tienen que ver con, atención presencial, preferencia del material impreso (libro) y socialización, seguido por el conocimiento y el no tener acceso a dispositivos electrónicos así como a internet. Estas últimas, muestran más una condición que presenta la población, que una clara preferencia según su experiencia en la modalidad a distancia.
Preferencia de los medios y materiales didácticos: Se presenta como material didáctico de preferencia de la población el material escrito impreso (60\% y $68 \%)$ al ubicarlo como el primer material en orden de preferencia, manteniéndose la tendencia a su vez del formato impreso $(43 \%$ y $39 \%)$. Seguido del material digital y el medio la plataforma virtual.

En la preferencia del formato digital, prevalecen razones que permiten más bien el uso de otros dispositivos electrónicos, asignándole por tanto, la posibilidad de ampliar los temas en estudio mediante "enlaces de interés", lo que la población asume como ventaja al considerar "los videos y audios potenciadores (ayudan) en la comprensión de la o las temáticas en estudio. Además de establecer como ventaja, la posibilidad de variar, en el caso de requerirse, características de formato, como el tamaño de la letra.

En el caso de la población que indica preferir ambos formatos ( $48 \%$ y $50 \%$ ), prevalecen aquellas características o posibilidades indicadas anteriormente según sea el formato. En el caso de los materiales digitales se indica, que permiten ampliar o complementar las temáticas, como la razón "los videos y audios ayudan a la comprensión de la temática", también la posibilidad de poder accesar a estos por diferentes dispositivos electrónicos, "ofrecen enlaces de interés para ampliar los temas" y destacan del formato impreso, las que permiten el contacto físico con el material como "subrayar y hacer anotaciones, además de la "resolución de ejercicios" y la "lectura continua".

Participación y comunicación: La mayoría (91\% y 73\%) de la población estudiantil afirma no participar en grupos (factor tiempo), pero sí considera de importancia (78\% y $88 \%$ ) la participación como parte de la vida universitaria, asignándole desde su experiencia ventajas no solo académicas, sino también sociales de formación integral, socialización, beneficio para la comunidad, vinculación con la institución y espacio recreativo.

Prefieren el uso de medios sincrónicos de comunicación que les permita mantenerse en contacto y con información relevante y a tiempo.

Es una población que evidencia tomar la iniciativa para establecer una interacción con sus pares, ya que manifiestan coordinar con otros estudiantes espacios de comunicación e intercambio de información y acompañamiento en el proceso de aprendizaje. Esta además, valora la interacción con otros y la promueven según sus necesidades. 


\section{DISCUSION}

Dentro del ámbito de la Educación a distancia, se considera generalmente que la población estudiantil es más heterogénea (García, 2014), situación que se presenta en algunas características de esta variable, en aspectos de importancia sociodemográfica, como la edad (jóvenes y jóvenes adultos, grupo promedio más representativo 29 años) y el sexo, (se confirma nuevamente la mujer como la mayor representación estudiantil). Sin embargo, al ser la población de modalidad a distancia y conociendo que la misma fundamenta su accionar en una población diversa, es necesario que se considere no solo a sus mayorías, sino poblaciones con una etapa social distinta, tal es el caso de la población de estudiantes en edad adulta (por encima de los 35 años), siendo por tanto, una población de interés institucional.

Hay una tendencia cada vez menos del estudiante convencional dedicado a tiempo completo al estudio (Soler, 2013), en este caso sin embargo se evidencia una población, que podría tener una dedicación de tiempo considerable al estudio, ya que indica no trabajar, aunque se desconoce las razones (que pueden ser multicausales), de la no integración a la fuerza laboral. Sin embargo queda claro, que se elige la modalidad por la oportunidad de llevar los estudios y el trabajo u otras actividades de índole personal, de forma paralela con las responsabilidades que esto conlleva.

A pesar de las condiciones anteriores, la población indica interrumpir los estudios universitarios en un porcentaje alto por razones que giran en torno en su mayoría a situaciones económicas o de índole laboral, condiciones circunstanciales que podrían estar limitando la continuidad y, por tanto, la permanencia y concreción de la formación profesional en la población.

Si bien es cierto se evidencia que la población en un porcentaje alto (65\%), tiene no solo dispositivos electrónicos, sino también una herramienta (internet) que los optimiza, las horas de uso son limitadas (si se piensa que estas no son necesariamente para el área académica), en promedio la mayoría de la población usa internet 2 horas al día para asuntos de diversa índole. Es necesario considerar en este punto, que se estén presentando situaciones circunstanciales (Tancredi, 2011), que limiten su optimización en la formación profesional, desde situaciones propias de la población estudiantil, hasta situaciones externas como en la oferta académica, el nivel académico que cursa, la carrera y su naturaleza disciplinar, las asignaturas, entre otros, por lo que se considera necesario investigar esta variable en específico.

En cuanto a preferencia de las asignaturas se opta por aquellas que, en su proceso de formación profesional, tengan algún componente virtual (híbridas). Se evidencia, por lo tanto, que la población prefiere aquellas experiencias de formación profesional que contemplen lo virtual o bien lo híbrido sin irse a los extremos de lo totalmente virtual o lo regular (sin componentes virtuales), tendencia que se evidencia en literatura reciente.

Es importante indicar que la población al elegir, ya sea la modalidad virtual o bien regular, en ambas se observan razones que dan pie a una serie de condiciones o circunstancias, como el tener o no los dispositivos o herramientas tecnológicas, que parece supeditan la preferencia manifiesta por la población. Por lo que cabe la interrogante, de no existir estas condiciones externas, ¿se mantendría esta preferencia en la población que indica elegir la modalidad regular?

La población prefiere la posibilidad de tener materiales en ambos formatos en su proceso formativo; sin embargo, cuando se compara la preferencia entre material impreso y digital, el impreso logra sobre pasar al digital. Por lo que se podría considerar según la experiencia de la población estudiantil, al primero como imprescindible en el proceso de aprendizaje en la modalidad a distancia. Sin embargo, al presentarse población que indica preferir ambos formatos, también se evidencia la necesidad de complementar la propuesta de materiales didácticos con aquellos que aportan otras formas de acceder a la información objeto de conocimiento. Se demuestra así, que la población estudiantil presenta una apertura ante las posibilidades tecnológicas en su proceso de formación profesional, a pesar de que hay población que pueda no tener los dispositivos o la competencia informática (Morales \& Espinoza, 2003).

Es evidente que la población presenta una marcada predilección por la coexistencia de materiales y medios, según sus condiciones o preferencias al tener la posibilidad de contar con las potencialidades de ambos formatos, lo que podría ser una oportunidad para establecer propuestas más flexibles a partir de las preferencias personales (Iriondo \& Gallego 2013), en lugar de tener propuestas generales, permitiendo que cada estudiante asuma un protagonismo en su proceso de aprendizaje.

Finalmente, en relación a la participación y comunicación la población estudiantil expresa que no acostumbra participar en las diferentes actividades extrauniversitarias que ofrece la universidad, pero consideran estas como parte importante de una comunidad universitaria.

En cuanto a la comunicación por medio de las tecnologías entre la población estudiantil, estos, buscan la manera de mantenerse en contacto por iniciativa propia más que como una opción que ofrezca o impulse la universidad. El Apéndice Digital 2 presenta una lista de conclusiones. 
Consideramos que la interrupción de los estudios en la población estudiantil tema de interés institucional, ya que se presenta una cantidad importante de población que evidencia la suspensión del proceso educativo al menos por dos periodos académicos, situación que va en detrimento no solo de los tiempos de conclusión de estudios, sino de la concreción de su formación profesional. La Universidad debe tomar acciones en procura para minimizar esta tendencia.

El formato impreso se mantiene como el material didáctico de preferencia, por lo que debe prevalecer el interés por conocer y comprender las preferencias de los estudiantes, en cuanto a las posibilidades de este, aunado a las potencialidades del material digital, ya que se evidencia una población estudiantil que aboga por la coexistencia de formatos y tipos de materiales, así como la apertura por tener en su proceso de aprendizaje las ventajas de la virtualidad.

Es necesario dados los resultados de este estudio, indagar específicamente criterios, motivaciones y posibilidades reales de participación de la población estudiantil en los grupos que organiza la Universidad, así mismo indagar sobre las diferentes posibilidades en que se puede incursionar en espacios comunicativos de manera que se fortalezcan lazos entre la población estudiantil y la universidad y se vea robustecida la formación profesional.

Es importante fortalecer los CEU como punto de encuentro desde una perspectiva integral, tanto en su dimensión académica, administrativa y de infraestructura, como en el acompañamiento y orientación que se pueda brindar a la población estudiantil, que permita establecer diferentes estrategias de apoyo y acercamiento durante el proceso formativo.

Analizar el uso y la capacidad de respuesta que tiene el CEU a las consultas de la población estudiantil, de manera que las dudas y consultas sean atendidas oportunamente, desde cualquier medio.

\section{AGRADECIMIENTOS}

Agradecemos el apoyo de las personas encargadas de los Centros Universitarios: Evelyn Gutiérrez, Sindy Scafidi, Annia Quesada. A la encargada de la Cátedra de Informática Educativa: Gabriela Bejarano S. A la encargada del Programa de Orientación y Atención Psicoeducativa: Ligia Arguedas R. de la Dirección de Tecnología, Información y Comunicaciones; Israel Azofeifa R. De la Vicerrectoría de Investigación: Ligia Bermúdez M. Al grupo de estudiantes que participaron en la validación y complete del instrumento de investigación.

\section{REFERENCIAS}

Fernández, N. (2012). Retención y Persistencia Estudiantil en Instituciones de Educación Superior: Una Aproximación Interdisciplinaria al Concepto. Paradigma, 33(2), 63-88. Recuperado de: http://www.scielo.org.ve/scielo.php?pi$\mathrm{d}=$ S1011-22512012000200004\&script=sci_arttext

García, L. (2012). Principios pedagógicos clásicos en el currículo, también en educación a distancia. Las nuevas fronteras de la educación a distancia. Secretaría Ejecutiva del CALED, Virtual Educa a través del Observatorio de la Educación Virtual en América Latina y el Caribe y la Universidad Técnica Particular de Loja. Recuperado de http://virtualeduca.org/documentos/observatorio/oevalc_2012_(fronteras).pdf

García, L. (2014) Bases, mediaciones y futuro de la educación a distancia en la sociedad digital. Madrid, España: Editorial Síntesis.

Iriondo, W., \& Gallego, D. (2013). El currículo y la educación a distancia. RIED. Revista Iberoamericana de Educación a Distancia, 16(1), 109-132. Recuperado de http://www. redalyc.org/articulo.oa?id=331427377006

La Gaceta. (2002, 19 de mayo). Ley General de la Persona Joven. N 8261. Diario oficial la Gaceta de Costa Rica, San José, Costa Rica.

Méndez, V., Monge, J., \& Montero, M. (2000). Aplicaciones educativas de los multimedios: la perspectiva de quienes han recibido capacitación sobre el uso de la herramienta. Centro de Investigación Académica (CIAC), San José, Costa Rica: UNED.

Mirsirlis, G. (2009) Deudas y desafíos en la educación de jóvenes y adultos: una mirada desde un enfoque político - didáctico. Instituto para el desarrollo y la innovación educativa, OIE. Recuperado de: http://www.oei.org.py/idie.v2/pdf/ EPJA-Una_mirada_politico-didactica.pdf

Morales, O., \& Espinoza, N. (2003). Lectura y escritura: coexistencia entre lo impreso y lo electrónico. Educere, 7 (22) 213-222. Recuperado de http://www.redalyc.org/articulo.oa?id=35602211

Soler, I. (2013). Los estudiantes universitarios: perfiles y modalidades de vinculación con el estudio en la universidad española. (Tesis doctoral, Universidad de Valencia). Recuperado de: http://roderic.uv.es/bitstream/ handle/10550/30171/TESIS\%20INES\%20SOLER. pdf? sequence $=1$

Tancredi, B. (2011) Apuntes para resignificar la educación a distancia. RIED. Revista Iberoamericana de Educación a Distancia, 14 (1) 55-72. Asociación Iberoamericana de Educación Superior a Distancia, Madrid, Organismo Internacional. Recuperado de http://www.redalyc.org/ articulo.oa?id=331427214004

See Digital Appendix at: / Ver Apéndice digital en: http://investiga.uned.ac.cr/revistas/index.php/cuadernos 